\title{
GLAD!
}

Revue sur le langage, le genre, les sexualités

09 | 2020

Traductologies féministes

\section{Traduire en féministes}

Translating as Feminists

Vanina Mozziconacci, Héloïse Thomas et Samantha Saïdi

\section{(2) OpenEdition}

Journals

Édition électronique

URL : http://journals.openedition.org/glad/2428

DOI : $10.4000 /$ glad.2428

ISSN : 2551-0819

Éditeur

Association GSL

Référence électronique

Vanina Mozziconacci, Héloïse Thomas et Samantha Saïdi, «Traduire en féministes », GLAD! [En ligne], 09 | 2020, mis en ligne le 20 décembre 2020, consulté le 20 janvier 2021. URL : http://

journals.openedition.org/glad/2428; DOI : https://doi.org/10.4000/glad.2428

Ce document a été généré automatiquement le 20 janvier 2021.

\section{(c) (i) (9)}

La revue GLAD! est mise à disposition selon les termes de la Licence Creative Commons Attribution Pas d'Utilisation Commerciale - Pas de Modification 4.0 International. 


\title{
Traduire en féministes
}

\author{
Translating as Feminists
}

Vanina Mozziconacci, Héloïse Thomas et Samantha Saïdi

1 Ce dossier accueilli par GLAD! est l'un des fruits du projet de recherche FELiCiTE (Féminismes en Ligne : Circulations, Traductions, Éditions) initié à Lyon en septembre 2018. FELiCiTE a été créée suite à la participation de deux des cofondatrices aux ateliers «La Fabrique des humanités » organisés par l'association ATLAS et l'EHESS en 2017. Ce programme visait à «favoriser les traductions entre anglais et français dans le champ de la recherche en philosophie et sciences humaines et sociales et apporter à de jeunes chercheures un approfondissement de leurs compétences en traduction ${ }^{1}$ ». Les ateliers proposés dans ce cadre nous ont en effet permis de prendre la mesure des enjeux de traduction pour les SHS en général et pour les études de genre en particulier. FELiCiTE est ainsi née du constat que les questions de genre restent sous-problématisées dans les études de traduction, et du désir de penser une traductologie spécifiquement féministe.

Depuis sa création, FELiCiTE a organisé une trentaine d'événements. Son séminaire inspiré du programme de traductologie féministe proposé dès 1988 par Lori Chamberlain $(1988$, p. 471 ; §44) - aborde des questions aussi variées que l'inscription $\mathrm{du}$ genre grammatical, la désignation sémantique des femmes ou du féminisme en langues arabe, chinoise, anglaise, la traduction et la circulation des idées féministes dans les mondes arabes et chinois, la place sociale des traductrices et éditrices de SHS dans le milieu académique ou encore la place de la traduction dans le champ postcolonial. Ce séminaire est également l'occasion de conférences et rencontres internationales, comme celle avec le collectif d'auteurices du Routledge Handbook of Translation, Feminism and Gender dirigé par Luise von Flotow et Hala Kamal (2020). Les ateliers de traduction sont également organisés autour de plusieurs langues, où nous invitons des spécialistes à réfléchir à partir de textes issus de corpus féministes fondamentaux; cela a donné lieu à des ateliers abordant l'arabe, l'allemand, l'italien, l'anglais, le serbo-croate et le chinois au sein desquels les masterante-'s du Master TLEC (Traduction Littéraire et Édition Critique) de Lyon 2 et du Master Études Italiennes de l'ENS de Lyon sont particulièrement actives et actifs. Ancrant le projet dans le chantier transversal Humanités Numériques du laboratoire Triangle (UMR 5206), nous avons 
organisé une journée Humanités Numériques et Traduction en avril 2018 en vue de dialoguer avec des concepteur.ices et développeureuses de plateformes de traduction collaborative ${ }^{2}$ afin d'évaluer la pertinence d'un développement dédié à FELiCiTE. Toujours dans cette perspective, nous avons également initié plusieurs collaborations fructueuses, comme celle avec le projet Transiens avec qui nous avons expérimenté la pratique de synthèses multilingues entre juin et septembre $2020^{3}$. Parmi d'autres actions plus ponctuelles de FELiCiTE, on peut également mentionner le suivi de stagiaire(s), l'acquisition d'ouvrages pour alimenter un fonds «Triangle (Genre, féminismes et traduction)» au sein du Centre de Documentation Recherche l'ENS de Lyon, le soutien à la publication pour des travaux impliquant en partie FELiCiTE, mais aussi et surtout des traductions originales proposées par nos membres et rendues disponibles en ligne ${ }^{4}$.

Bien que FELiCiTE comporte plusieurs volets, la problématique centrale qui noue les différentes recherches menées en son sein reste la traductologie féministe - au sens d'une approche féministe de la traductologie, de la traduction en sciences humaines et sociales, et de l'étude des traductions des textes féministes. Il s'agit d'inscrire la traduction au cœur de pratiques de recherche en études de genre. Cela peut par exemple se faire à travers l'analyse des rapports entre femmes et traduction depuis l'enjeu plus général du rapport des femmes au savoir. En effet, historiquement, les institutions scolaires et universitaires accordent à ces dernières une place spécifique et subalterne, davantage centrée sur le droit de recevoir et de passer des savoirs que sur celui d'en produire - comme le montrent les chiffres rapportés dans les études sociologiques et politiques de Rogers et Molinier (2016) ou de Marry et al. (2017). Cette place étriquée est décrite pour l'histoire française par Nicole Mosconi au travers de la distinction entre « sachantes » et « savantes ». Ces deux figures caractérisent le rapport au savoir des femmes en tant qu'il est ou non autorisé : il est à présent considéré comme normal que des femmes enseignent et soient donc " transmetteuses de savoir ", mais l'idée qu'elles puissent être également "productrices et créatrices de savoirs nouveaux » est loin d'être admise (2004). Si les femmes sont aisément perçues comme "studieuses", voire "sachantes", elles sont plus rarement considérées comme «savantes" (Mosconi, 1990). Mosconi reprend à Colette Audry l'idée selon laquelle les femmes seraient assignées au statut $d^{\prime}$ ' "usufruitières » et non d'« héritières de plein droit » dans le domaine scientifique. Si l'accès des femmes à l'instruction a donc été une inclusion faisant suite à une exclusion, cela a constitué une égalité qui s'est avérée plus formelle que réelle. De plus, cette entrée dans les institutions du savoir a également représenté un recul à d'autres égards, une forme d'expropriation ou d'exil: «En accédant à l'instruction, elles ont été en partie expatriées de leurs propres expériences et de leurs propres savoirs puisque rien ou peu de choses dans les savoirs qu'elles acquéraient [ne] leur permettait de comprendre et de théoriser leur propre expérience, leur propre situation sociale, leur propre place dans les rapports sociaux de sexe" (Mosconi, 1994, p. 83). Assigner aux femmes la transmission d'une culture à garder intacte, en faire des consignataires d'un patrimoine (qui porte bien son nom) et non des sujets novateurs ou transformateurs, revient à les placer, une fois encore, du côté de la conservation et de la reproduction.

4 Si l'on garde à l'esprit cette ambivalence dans la construction historique du rapport au savoir des femmes, on peut imaginer qu'on attend des traductrices, plus que des traducteurs (adeptes des Belles Infidèles), une forme de révérence vis-à-vis du texte source, une invisibilisation de soi qui conduirait à (prétendre) laisser parler le texte de 
lui-même. Le rapport genré aux discours savants conduirait à ce que les traductrices soient "toutes ouïe", respectueuses, voire timides, face au discours de l'autre," comme si elles faisaient confiance au texte pour dire lui-même son sens » (Michèle Le Dœuff, 1980, p. 161). Une telle idée trouverait écho dans les métaphores sexistes et sexuelles qui peuplent la tradition traductologique dominante, comme celles de l'appropriation sexuelle et coloniale ou celles qui opposent beauté et fidélité en traduction, métaphores analysées avec beaucoup de finesse dans le texte de Lori Chamberlain (traduit par Samantha Saïdi) à retrouver dans ce numéro. Le traducteur doit-il commettre un acte de séduction ou d'agression pour s'approprier le texte? Et la traductrice doit-elle rester fidèle? Et cette fidélité implique-t-elle une forme d'invisibilisation de soi, là où la beauté s'accompagne toujours du soupçon du narcissisme? Traduire en féministe, en s'appuyant en particulier sur les épistémologies du standpoint - dites du positionnement ou du point de vue (Puig de la Bellacasa, 2012) - impliquerait de contrarier ce modèle « féminin » de la traduction qui associe fidélité et effacement de soi. Ainsi, comme l'ont conceptualisé, dès les années 1990 au Canada, des spécialistes de la traduction de textes féministes (de l'anglais vers le français et vice versa) comme Susanne de Lotbinière-Harwood (Lotbinière-Harwood 1991), la traduction permet à la fois une réappropriation des concepts de la langue source et l'affirmation d'une langue féministe face au discours patriarcal et androcentré dans la langue cible. Retravaillant l'opposition dénoncée par Lori Chamberlain (\$35-37) entre travail « créatif » ou "productif » de l'écriture et « re-créatif » ou « reproductif » de la traduction, nous affirmons ici que le travail de traduction est lui-même, au moins indirectement, un travail productif. C'est ainsi que Cornelia Möser, dans le texte intitulé « Traduire c'est produire » qu'elle publie dans ce numéro, rappelle que l'idéal du texte traduit qui serait un "calque » de l'original est à bannir, car c'est ce même idéal qui conduit à des crispations autour de l'idée qu'il existe des intraduisibles censés manifester des frontières infranchissables. S'en défaire permet également de dissiper l'illusion de neutralité qui accompagnerait une représentation de la traduction comme réplique indifférente aux contextes. En opposition à ces deux écueils, le féminisme est une bonne occasion selon l'autrice de considérer la traduction comme un vecteur essentiel et transversal dans la circulation des idées politiques et des sciences humaines et sociales.

5 La question n'est donc pas seulement de traduire en féministe mais de traduire les féminismes. Par exemple, la question se pose autour du concept féministe de care, dont la non-traduction est parfois perçue comme une concession au "globish", ce Global English hégémonique qui [...] vient standardiser les discours et les sentiments » (Ibos, Damamme, Molinier, Paperman, 2019, p. 19). Il s'agit plutôt de le saisir comme un « intraduisible » au sens où Barbara Cassin définit ce terme, à savoir « non pas ce qu'on ne traduit pas, mais ce qu'on ne cesse pas de (ne pas) traduire » (2014). À travers l'usage de la notion, ce n'est pas seulement un mot mais bien un travail qu'il s'agit de redéfinir : cet enjeu s'est retrouvé au sein des théories économiques des « cuidados ${ }^{5}$ » en Espagne, sur lesquelles porte un texte collectif (Andrea Barbara Blazsek, Maria Celeste Linardelli, Daniel Garcia, Maria Agustina Diez) traduit par Charlie Brousseau dans ce numéro. Nous retrouvons là l'idée de "circulation", si importante dans le projet FELiCiTE, impliquant des allers-retours entre les courants, entre les ères et les aires. Ce phénomène est également illustré par l'article de Léa Buatois dans le présent dossier, qui aborde la traduction des textes de He-Yin Zhen - féministe anarchiste chinoise née à la fin du XIX siècle. L'autrice montre que le travail qui consiste à «rendre en 
français » le lexique de He-Yin Zhen implique de retracer en quoi le texte source de la féministe est lui-même, relativement à d'autres références, un texte cible (qui reprend par exemple du vocabulaire issu de traductions de Mikhaïl Bakounine en chinois). Bien que les outils qui s'y trouvent ne soient donc pas tous des créations originales, c'est au travers d'expressions nouvelles (mêlant terminologie marxiste et souci féministe) que s'élabore un lexique inédit.

6 Les traductrices féministes préfèrent-elles alors trahir que traduire, pour inventer, créer, subvertir? Pour reprendre la question posée par Touriya Fili-Tullon, Oristelle Bonis, Roula Sadiki et Souad Labbize en sommes-nous à "envisager une symétrie (totale) entre le geste créatif de l'écriture, traditionnellement mis du côté du masculin, et celui de la "copie" attribuée à la traduction et à la relecture, placées habituellement du côté du féminin »? Pour ne pas en arriver à une telle assimilation, fautive, il importe de replacer le travail de traduction dans sa réalisation concrète, en particulier dans sa dimension éditoriale. Revenant sur l'entreprise de traduction en arabe du texte Enjamber la flaque où se reflète l'enfer. Dire le viol, témoignage poignant de Souad Labbize dédié aux victimes d'agressions sexuelles, les traductrices Touriya Fili-Tullon et Roula Sadaki rappellent que c'est également une relation faite de mémoire intertextuelle, de sensibilité féministe commune et de solidarité transnationale qui a permis de donner à entendre "une subjectivité libérée de quarante ans de silence». La traduction commence d'abord par une volonté de diffusion - c'est en cela qu'elle doit être pensée, non in abstracto mais au sein même du processus d'édition - et par la conviction de la nécessité de faire porter (davantage) une voix.

7 En réalité, c'est moins le parti-pris ou la contingence qui caractérise l'approche féministe de la traduction que la revendication même de choix ou le refus d'une affirmation de neutralité, considérée comme illusoire (refus que les traductrices féministes ne sont pas les seules à revendiquer [Eco, 2006]). La traduction, parce qu'elle est un discours produit dans certaines conditions et depuis certaines coordonnées sociales, est, elle aussi, "située». Pas moins neutre qu'une autre, la traduction féministe se reconnaît quant à elle comme n'écrivant pas «depuis nulle part » et n'allant pas "partout». On pourrait en ce sens reprendre la déclaration de Christine Delphy, selon laquelle " [T]oute connaissance est le produit d'une situation historique, qu'elle le sache ou non. Mais qu'elle le sache ou non fait une grande différence; si elle ne le sait pas, si elle se prétend "neutre", elle nie l'histoire qu'elle prétend expliquer, elle est idéologie et non connaissance " (Delphy, 1998, p. 265). Lorsque la traduction se prétend neutre, c'est-à-dire si elle ne reconnaît pas la part de décision ${ }^{6}$, d'invention et de transformation qui la constitue, elle transmet des représentations clandestines parfois parasites et charrie des impensés du traducteur ou de la traductrice, comme peut le faire un curriculum caché. La chercheuse en lettres classiques Emily Wilson, première femme à avoir traduit l'Odyssée d'Homère en anglais (2017) - et à laquelle est consacrée la belle bande dessinée « Les sirènes de l'interprétation » d'Elsa Abderhamani et Julia Burtin Zortea, présentée dans ce numéro - rappelait récemment que beaucoup de traducteurs avaient intégré au texte des termes sexistes ou misogynes qui n'existaient pas dans le grec originel.

8 L'attention portée par Wilson aux questions de genre l'a également conduite à ne pas atténuer la puissance des personnages féminins - déesses, femmes sans droits ou femmes esclaves - dans l'odyssée. Ainsi, au moment où Pénélope déverrouille la réserve dans laquelle Ulysse entrepose ses armes, Homère décrit ses mains avec le terme 
«pakhus », qu'on retrouve dans « pachyderme », et qui signifie « épais ». Or, cet attribut étant considéré comme peu féminin par les autres traducteurs, plusieurs d'entre eux ont supprimé l'adjectif ou, comme Robert Fagles (dont la traduction date de 1996), ont utilisé un terme euphémisé pour la traduction anglaise, tel que "steady» (ferme/ stable). Wilson, quant à elle, a traduit le passage ainsi : « Her muscular, firm hand / picked up the ivory handle of the key " ", arguant que le tissage a effectivement pour conséquence de muscler les mains. Elle ajoute: "Je voulais être sûre que ma traduction, comme l'original, souligne les capacités physiques de Pénélope, qui la caractérisent comme un personnage qui joue un rôle crucial dans l'action - et ce, qu'elle connaisse ou non les implications de ce qu'elle fait " (North, 2017, en ligne). En refusant de passer sous silence certains aspects dans les descriptions de Pénélope, Wilson cherche à ne pas minimiser la puissance d'agir du personnage. Mais cela ne signifie pas pour autant que la traductrice revendique avoir proposé une traduction finale, anhistorique et universelle :

Il n'y a jamais de traduction définitive et une traducteur/rice fait toujours des choix. Ce à quoi je m'oppose, c'est au fait que les gens tendent à considérer d'autres traductions en se disant "ils n'ont pas fait de choix, ils ont simplement écrit ce qu'il $\mathrm{y}$ avait dans la langue grecque". Or, tout le monde fait des choix, et celles et ceux qui traduiront dans cent ans feront aussi des choix. Par ailleurs, le regard que nous portons sur un texte donné d'une période particulière antérieure est informé par notre propre culture. (2018, en ligne)

9 La traduction de Wilson étant très différente d'autres traductions antérieures, il lui a été reproché d'avoir produit un texte biaisé, voire un texte faisant passer un message féministe anachronique qui n'existerait pas dans l'original. Or, les exemples précédents révèlent que les «lunettes du genre " - pour reprendre l'expression d'Isabelle Clair (2015) - chaussées par Wilson ne la conduisent pas à injecter du féminisme là où il n'y en aurait pas, et qu'elle ne cherche pas même à "tordre le bâton dans l'autre sens " face à des traductions sexistes. L'ironie est que la démarche de traduction accusée d'être biaisée est celle qui cherche justement à restituer des aspects occultés dans l'original ou à corriger des sur-traductions: le prisme féministe s'avère en réalité heuristique pour redécouvrir le texte. Le fait qu'elle soit à contre-courant d'une tradition ne constitue pas la preuve qu'elle engage une démarche plus partiale que les autres traductions; c'est même le contraire, si on suit celui qui a justement théorisé l'idée de Wertfreiheit, "neutralité axiologique " (aussi traduit par "non-imposition de valeurs ») en sciences, à savoir Max Weber. Ce dernier rappelle qu'un positionnement à contre-courant peut être l'occasion de pointer comme douteux ce qui était devenu une évidence :

[I]l n'y a pas de doute qu'un anarchiste peut être un bon connaisseur du droit. Et s'il l'est, le point archimédien, pour ainsi dire, où il se trouve placé en vertu de sa conviction objective - pourvu qu'elle soit authentique - et situé en dehors des conventions et des présuppositions qui paraissent si évidentes à nous autres, peut lui donner l'occasion de découvrir dans les intuitions fondamentales de la théorie courante du droit une problématique qui échappe à tous ceux pour lesquels elles sont par trop évidentes (1965, en ligne).

C'est la réflexivité qui accompagne la situation et les orientations d'une traduction qui rendent celle-ci robuste et susceptible d'être jugée avec objectivité. Cela passe par un effort d'élucidation, effort dans lequel la traductrice ou le traducteur s'engage, et, forcément, s'expose. La posture de retrait qui prétend laisser intact - au sens où l'on ne toucherait pas - le texte peut conduire à jeter celui-ci en pâture à des mésusages ou à 
des instrumentalisations. C'est ce que montre Samantha Saïdi dans son présent article, qui aborde d'un point de vue linguistique des formes d'ambiguïté (liées au genre, mais pas seulement) des typologies produites par les études sur les violences de couple (telles que «intimate partner violence» ou "lesbian battering»). S'il ne s'agit pas de résoudre des imprécisions qui seraient volontaires ou entretenues par des auteurs/ rices, l'enjeu est néanmoins, pour les textes que l'autrice aborde, « d'éviter de suggérer dans les énoncés produits en français une bidirectionnalité ou essentialisation genrée des violences lorsqu'elles sont absentes en anglais ». Autrement dit, si le mot-à-mot produit une équivoque, une obscurité, qui apparaît par le processus même de translation d'une langue à l'autre, alors il est de la responsabilité de la traductrice ou du traducteur de la tirer au clair. Dans le cadre d'analyse secondaire en sciences sociales, cette responsabilité d'élucidation revient à ceux ou celles qui font ré-usage des typologies ou concepts produits par d'autres.

11 Pour toutes ces raisons, dans une perspective féministe, il est pertinent de considérer la traduction comme un effort itératif permanent vers l'interprétation et la transmission, en la replaçant au sein de la tradition herméneutique (Ricœur 2004 ; Wilhelm 2014). On pourrait alors s'étonner que le rapprochement entre cette dernière et les théories féministes soit, historiquement, loin d'aller de soi. C'est ce paradoxe qu'explore l'article d'Axelle Cressens, qui met au jour les rendez-vous manqués et les malentendus qui semblent caractériser la relation entre ces deux (écoles de) pensées. Pour dépasser cela, elle dessine les contours d'une herméneutique féministe qui combinerait justesse de l'interprétation et justice interprétative. Ce faisant, elle souligne le lien étroit entre l'enjeu politique et l'enjeu épistémique dans toute démarche qui vise à critiquer les fondements androcentriques et eurocentriques des théories formalisant l'interprétation des signes.

12 Suivant l'esprit invoqué dans les articles, ce dossier revendique non l'exhaustivité ou la finalité dans ses analyses, mais la multiplication des regards portés sur les relations entre traduction et féminisme. Refusant d'avoir le «mot de la fin », les traductologies féministes déstabilisent, voire renversent, les paradigmes traductologiques implantés qui, arrimés à une conceptualisation genrée de la création et du langage, hiérarchisent et dépolitisent le processus de traduction. Elles déjouent frontières, censures ou notions d'intraduisibles pour libérer les idées des empêchements patriarcaux et coloniaux. Il ne s'agit même plus de subvertir les notions de fidélité, d'objectivité, ou de genre grammatical par exemple, mais bien de les refonder en les désenclavant et en les recontexualisant, c'est-à-dire en les réinscrivant dans des réseaux de création et de circulation des idées, et en les articulant à une approche pluridisciplinaire du langage et des textes. La pluralité des traductologies féministes les conduit également à réintégrer une forte dimension éthique et politique, non en imposant une grille d'analyse particulière mais en faisant ressortir les instabilités et ambiguïtés sémantiques originelles, cela afin de soustraire le texte à certains carcans herméneutiques hégémoniques et de favoriser la multiplicité des lectures. La traduction, dans le cadre d'une théorie et d'une pratique féministe, n'aboutit plus à un résultat statique et figé mais reste alors un processus dynamique exponentiel, voire fondamentalement émancipateur. 


\section{BIBLIOGRAPHIE}

CHAMBERLAIN, Lori, 1988, « Gender and the Metaphorics of Translation ». Signs, vol. 13, no. 3, pp. 454-472. JSTOR, www.jstor.org/stable/3174168. Accessed 16 Dec. 2020.

CHANNEL 4 NEWS, 20 mai 2018, « A translator always makes choices. Has The Odyssey been mistranslated? » https://www.facebook.com/watch/?v=10155936220521939.

CLAIR, Isabelle, 2015, Sociologie du genre, Paris, Armand Colin.

DELPHY Christine, 1998, L'ennemi principal 1. Économie politique du patriarcat, Paris, Syllepse.

ECO, Umberto, 2006, Dire presque la même chose - Expériences de traduction, Paris, Éditions Grasset et Fasquelle, traduit de l'italien par Myriem Bouzaher.

FLOTOW, Luise von et KAMAL, Hala (dir.). 2020. The Routledge Handbook of Translation, Feminism and Gender. New York : Routledge.

HOMER, 2017, The Odyssey, New York: WW Norton \& Co, traduction du grec par Emily Wilson.

LE DEEUFF Michèle, 1980, L'imaginaire philosophique, Paris, Payot.

LOTBINIÈRE-HARWOOD, Susanne, de, 1991, Re-belle et infidèle. La traduction comme pratique de réécriture au féminin. The Body Bilingual, Translation as a Rewriting in the Feminine. Montréal : Les éditions du remue-ménage/Women's Press.

MARRY, Catherine, BERENI, Laure, JACQUEMART, Alban, POCHIC, Sophie et REVILLARD, Anne. 2017. Le plafond de verre et l'état. Paris : Armand Colin.

MOSCONI, Nicole, 1990, « La femme savante [Figure de l'idéologie sexiste dans l'histoire de l'éducation] », Revue française de pédagogie, vol. 93, no 1, p. 27-39.

MOSCONI, Nicole, 1994, Femmes et savoir : la société, l'école et la division sexuelle des savoirs, Paris, L'Harmattan.

MOSCONI, Nicole, 2004, «Les femmes et les disciplines instituées ", in Intellectuelles : du genre en histoire des intellectuels, N. RACINE et M. TREBITSCH (dir.), Bruxelles, Belgique, Éditions Complexe.

NORTH, Anna, 2017, « Historically, men translated the Odyssey. Here's what happened when a woman took the job.», Vox.com : https://www.vox.com/identities/2017/11/20/16651634/ odyssey-emily-wilson-translation-first-woman-english

PUIG DE LA BELLACASA, Maria, 2012, Politiques féministes et construction des savoirs : « penser nous devons!", Paris, L’Harmattan.

RICCEUR, Paul, 2004, Sur la traduction. Paris, Les Belles Lettres.

ROGERS, Rebecca et MOLINIER, Pascale. 2016. Les femmes dans le monde académique : perspectives comparatives. Rennes : Presses universitaires de Rennes.

WEBER, Max, 1965 [1904-1917], Essais sur la théorie de la science, Julien Freund. Paris : Librairie Plon, traduit de l'allemand par Julien Freund. En ligne : http://classiques.uqac.ca/classiques/ Weber/essais_theorie_science/essais_theorie_science.html

WILHEM, Jane, 2014, « Anthropologie des lectures féministes de la traduction ». TTR : Traduction, terminologie, rédaction, 27(1), p.149-188. 


\section{NOTES}

1. https://www.atlas-citl.org/la-fabrique-des-humanites/

2. https://hypertopic.org/traduxio

3. https://bssg.hypotheses.org/749

4. Voir notamment: https://journals.openedition.org/glad/1647 et https:// reflexivites.hypotheses.org/11736 et dans ce numéro la traduction du texte de Lori Chamberlain : https://journals.openedition.org/glad/2057

5. Voir les recherches actuelles de Viviane Albenga: https://durkheim.u-bordeaux.fr/Notreequipe/Chercheur-e-s-et-enseignant-e-s-chercheur-e-s-associe-e-s/CV/Viviane-Albenga

6. Rappelons au passage que même les algorithmes des traductions automatiques font des choix, parfois sexistes, puisqu'ils sont le reflet des préjugés de ceux qui les programment.

7. «Sa main ferme et musclée / prit la poignée en ivoire de la clef ».

\section{INDEX}

Keywords : translation, gender, feminism, FELiCiTE

Thèmes : Recherches

Mots-clés : traductologie, traduction, genre, féminisme, FELICITE

\section{AUTEURS}

\section{VANINA MOZZICONACCI}

Vanina Mozziconacci est maîtresse de conférences en philosophie et en sciences de l'éducation à l'Université Paul Valéry Montpellier 3. Ses recherches portent plus particulièrement sur les théories féministes de l'éducation. Parmi ses dernières publications, on trouve " Faut-il être femme pour philosopher ?", La vie des idées [En ligne], 2020 et « "Le personnel est académique". Pour une subversion féministe de l'université, de la pédagogie à l'institution ", Genre, sexualité \& société [En ligne], 22 | 2019.

\section{HÉLOÏSE THOMAS}

Héloïse Thomas est doctorante en Études Américaines à l'Université Bordeaux Montaigne. Ses recherches portent sur les représentations de l'histoire et de la futurité dans la culture et la littérature nord-américaines du XXe et XXIe siècles, à travers un prisme féministe, queer et décolonial. Elle a récemment publié des articles sur la poésie de Robin Coste Lewis, la représentation lesbienne dans les séries contemporaines et la notion de home dans l'œuvre d'Alison Bechdel.

\section{SAMANTHA SAÏDI}

Samantha Saïdi est ingénieure d'études en Humanités Numériques et traductrice anglais-français en sciences humaines et sociales à l'ENS de Lyon, rattachée au laboratoire Triangle UMR5206. Après des études de linguistique anglaise (Nantes) et en sciences de l'information (ENSSIB), elle travaille à l'édition numérique de différents corpus de sciences humaines et sociales comme 
L'écho de la Fabrique, La Storia d'Italia, ou La bibliothèque Foucaldienne. Elle reprend en 2017 ses études en traduction et fonde avec Vanina Mozzicanacci, Françoise Orazi, Hélö̈se Thomas et Noémie Grunenwald le séminaire de traductologie féministe FELiCiTE et ses ateliers de traduction féministe en SHS (arabe, espagnol, italien, anglais). 